\title{
Article
}

\section{Temporal resolution of a pre-maximum halt in a Classical Nova: V5589 Sgr observed with STEREO HI-1B}

Eyres, Stewart Paul shore, Bewsher, Danielle, Hillman, Y, Holdsworth, Daniel Luke, Rushton, M T, Bresnahan, David William, Evans, A and Mroz, P

Available at http://clok.uclan.ac.uk/17142/

Eyres, Stewart Paul shore, Bewsher, Danielle ORCID: 0000-0002-6351-5170, Hillman, Y, Holdsworth, Daniel Luke ORCID: 0000-0003-2002-896X, Rushton, M T, Bresnahan, David William, Evans, A and Mroz, P (2017) Temporal resolution of a pre-maximum halt in a Classical Nova: V5589 Sgr observed with STEREO HI-1B. Monthly Notices of the Royal Astronomical Society . ISSN 0035-8711

It is advisable to refer to the publisher's version if you intend to cite from the work. http://dx.doi.org/10.1093/mnras/stx298

For more information about UCLan's research in this area go to http://www.uclan.ac.uk/researchgroups/ and search for <name of research Group>.

For information about Research generally at UCLan please go to http://www.uclan.ac.uk/research/

All outputs in CLoK are protected by Intellectual Property Rights law, including Copyright law. Copyright, IPR and Moral Rights for the works on this site are retained by the individual authors and/or other copyright owners. Terms and conditions for use of this material are defined in the policies page. 


\title{
Temporal resolution of a pre-maximum halt in a Classical Nova: V5589 Sgr observed with STEREO HI-1B
}

\author{
S. P. S. Eyres ${ }^{1 \star}$ D. Bewsher, ${ }^{1}$ Y. Hillman,${ }^{2}$ D. L. Holdsworth,${ }^{1}$ M. T. Rushton,${ }^{3,1}$ \\ D. Bresnehan ${ }^{1}$ A. Evans, ${ }^{4}$ P. Mróz ${ }^{5}$ \\ ${ }^{1}$ Jeremiah Horrocks Institute, University of Central Lancashire, Preston PR1 2HE, UK \\ ${ }^{2}$ Department of Geosciences, Raymond and Beverly Sackler Faculty of Exact Sciences, Tel-Aviv University, Tel-Aviv 69978, Israel \\ ${ }^{3}$ Astronomical Institute of the Romanian Academy, Str. Cutitul de Argint 5, 040557, Bucuresti, Romania \\ ${ }^{4}$ Astrophysics Group, Keele University, Keele, Staffordshire ST5 5BG, UK \\ ${ }^{5}$ Warsaw University Observatory, Al. Ujazdowskie 4, 00-478 Warszawa, Poland
}

Accepted XXX. Received YYY; in original form ZZZ

\begin{abstract}
Classical novae show a rapid rise in optical brightness over a few hours. Until recently the rise phase, particularly the phenomenon of a pre-maximum halt, was observed sporadically. Solar observation satellites observing Coronal Mass Ejections enable us to observe the pre-maximum phase in unprecedented temporal resolution. We present observations of V5589 Sgr with STEREO HI-1B at a cadence of $40 \mathrm{~min}$, the highest to date. We temporally resolve a pre-maximum halt for the first time, with two examples each rising over 40 min then declining within 80 min. Comparison with a grid of outburst models suggests this double peak, and the overall rise timescale, are consistent with a white dwarf mass, central temperature and accretion rate close to $1.0 \mathrm{M}_{\odot}, 5 \times 10^{7} \mathrm{~K}$ and $10^{-10} \mathrm{M}_{\odot} \mathrm{yr}^{-1}$ respectively. The modelling formally predicts mass loss onset at JD 2456038.2391 $\pm 0.0139,12 \mathrm{hrs}$ before optical maximum. The model assumes a main-sequence donor. Observational evidence is for a subgiant companion; meaning the accretion rate is under-estimated. Post-maximum we see erratic variations commonly associated with much slower novae. Estimating the decline rate difficult, but we place the time to decline two magnitudes as $2.1<\mathrm{t}_{2}($ days $)<3.9$ making V5589 Sgr a "very fast" nova. The brightest point defines "day 0" as JD 2456038.8224 \pm 0.0139 , although at this high cadence the meaning of the observed maximum becomes difficult to define. We suggest that such erratic variability normally goes undetected in faster novae due to the low cadence of typical observations; implying erratic behaviour is not necessarily related to the rate of decline.
\end{abstract}

Key words: novae, cataclysmic variables - stars:individual:V5589 Sgr

\section{INTRODUCTION}

Classical novae are interacting binary stars, in which a white dwarf (WD) accretes matter from a (usually) mainsequence-like companion, generally in a close orbit with a period of a few hours to days. This results in a build up of material on the WD surface, leading to thermonuclear runaways (TNRs) that cause an outburst, at ultraviolet (UV), optical and infrared (IR) wavelengths in a few hours, and the ejection of around $10^{-5}$ to $10^{-4} \mathrm{M}_{\odot}$ of matter at velocities of a few 100 to a few $1000 \mathrm{~km} \mathrm{~s}^{-1}$ (Warner 2008). Decline then follows over the course of weeks or months, with the time to drop 2 or 3 magnitudes from maximum related to the absolute magnitude via the maximum-magnituderate-of-decline relationship (MMRD; Downes \& Duerbeck 2000), for both Galactic and extra-galactic populations

^ E-mail: spseyres@uclan.ac.uk (SPSE)
(Darnley et al. 2006). Slower novae often show erratic variations and remain bright for longer times than is consistent for the MMRD, when considered in the light of independent distance measurements. The relationship is also not useful for novae that decline most rapidly, as the absolute magnitude becomes insensitive to decline time (e.g. Capaccioli et al. 1990).

Recently observations and theoretical developments have established that, prior to optical maximum X-ray and even gamma ray emission is seen (Ackermann et al. 2014). Due to the rate of ascent, the pre-maximum optical lightcurve has been only rarely observed, and at cadences of $1-2$ data points per day. Some show a pre-maximum halt (PMH) that had not been subjected to theoretical modelling.

Hounsell et al. (2010) presented four light-curves taken with the Solar Mass Ejection Imager (SMEI) down to time resolutions of $80 \mathrm{~min}$, showing the $\mathrm{PMH}$ to be ubiquitous in their sample. Hillman et al. (2014) have demonstrated that the form of these halts varies with the gross parameters of 
WD mass, WD central temperature, and the accretion rate from the companion.

Here we present data from the STEREO HI-1B camera for V5589 Sgr, following the outburst from the initial rise, through the optical peak to the point where it fades below detectability for this instrument. The cadence is around 40 min, making these the highest time-resolution observation of this phase to date. We discuss the rise phase, including the PMHs, in the context of the models of Hillman et al. (2014). We also contrast the post-maximum behaviour with much slower Classical Novae (CNe) and suggest that the empirical split in this behaviour between slow and fast novae is in part a function of the typical one day sampling of the light-curves.

\section{V5589 SGR}

V5589 Sgr (Nova Sgr 2012, PNV J17452791-2305213, coordinates J2000 174528.03 -23 05 22.7) was discovered on 2012 April 21.0112 UT (Sokolovsky et al 2012) at V 9.6, rising to a reported maximum of $\mathrm{V}=8.8$ (Korotkiy et al. 2012) by 2012 April 21.654 UT (CBAT 2015). Pre-discovery images detected the outburst on 2012 April 20.8403 UT down to $\mathrm{V}=10.2$ (CBAT 2015).

Early spectroscopy indicated high velocity ejecta in this nova, with line widths (FWHM) of $5600 \mathrm{~km} \mathrm{~s}^{-1}$ (Korotkiy et al. 2012) to $6500 \mathrm{~km} \mathrm{~s}^{-1}$ (Esipov et al 2012). Observations in X-ray (Sokolovsky et al 2012; Nelson et al 2012a) and radio (Nelson et al 2012a) wavelengths shortly after optical peak showed non-detections, and suggested no shocked component in the expanding ejecta at this early stage of the decline.

Swift UVOT detected the object on 2012 April 25.7 at a UVM2 brightness of $13.71 \pm 0.09 \mathrm{mag}$, fading rapidly from 13.8 to 13.5 over a 5975 s observation (Nelson et al $2012 \mathrm{~b}$ ). By 2012 May 10 hard X-ray emission was present, consistent with a column of $N(H)=(3 \pm 1) \times 10^{21} \mathrm{~cm}^{-2}$ and a plasma temperature of at least $2.7 \times 10^{8} \mathrm{~K}$; the UVM2 brightness was $16.0 \pm 0.2 \mathrm{mag}$ by this date (Nelson et al 2012b). Weston et al. (2016) argue that the radio and X-ray characteristics are consistent with high-speed, low-mass ejecta that accelerates over the first few weeks after outburst.

The nova has been observed by the OGLE survey since 2010 (Mróz et al. 2015). In the quiescent light-curve they found eclipses with a period of $1.59230(5)$ days, which they interpret as the orbital period. Such a relatively long orbital period suggests that the secondary is a subgiant. If the secondary fills its Roche lobe the orbital period can be used to estimate the radius of the secondary (see e.g. King 1989). Using Eq (2.3.10) of King we find that the secondary radius $R_{2} / R_{\odot} \sim 0.11 \mathrm{P}_{\mathrm{hr}} \sim 4.2 R_{\odot}$, confirming that the secondary is evolved. The last pre-eruption image was taken on 2012 April 20.3728 UT at $I=16.90$. These authors also note that with this longer period, the companion is likely to be a subgiant. Weston et al. (2016) come to the same conclusion.

\section{OBSERVATIONS}

The Solar TErrestrial RElations Observatory (STEREO) consists of two nearly identical spacecraft, in heliocentric or- bits, one ahead of the Earth (STEREO-A) and the other behind the Earth (STEREO-B) (Kaiser et al. 2008). The twin spacecraft were launched on 2006 October 26, and the separation between each spacecraft and the Earth has increased by approximately $22^{\circ}$ per year since achieving helocentric orbits.

The Sun Earth Connection Coronal and Heliospheric Investigation (SECCHI) instrument suite (Howard et al. 2008) on STEREO includes the heliospheric imagers (HI-1 and HI-2). The HI instruments have been used to observe a variety of variable stars including classical nova V5583 Sgr (Holdsworth et al. 2014), and long period variables (Wraight et al. 2012).

Data from the HI-1 instument on STEREO-B (HI-1B) are used in this paper. HI-1B has a field-of-view (FOV) of $20^{\circ}$, which extends from $\sim 4-24^{\circ}$ elongation, with a pixel size of $\sim 70$ arcsec, (Eyles et al 2009). The STEREO HI$1 \mathrm{~B}$ instrument's main passband covers the range from $600-$ $750 \mathrm{~nm}$, but also has contributions at $300-450 \mathrm{~nm}$ and from $900-1100 \mathrm{~nm}$ (see Fig. 6 of Bewsher et al. 2010)

The HI-1B images consist of 30 exposures of $40 \mathrm{~s}$ each, with a summed image cadence of 40 min (Eyles et al 2009). Each exposure is scrubbed of cosmic ray hits prior to summing (Howard et al. 2008). The summed images constitute the L0.5 FITs files which are available to download from several websites including the UK Solar System Data Centre $\left(\mathrm{UKSSDC}^{1}\right)$.

The L0.5 data have been prepared with the SSWIDL procedure, SECCHI_PREP. This routine applies the shutterless correction to the data, the large scale flatfield determined by Bewsher et al. (2010), and the pointing and optical parameter calibration of Brown, Bewsher \& Eyles (2009). It also identifies missing blocks and saturated pixels, filling these pixels with $\mathrm{NaN}$ values.

As discussed in Bewsher et al. (2010), the point spread function (PSF) of the HI-1B instrument is well approximated with a Gaussian profile, and the aperture size of 3.1 pixels suggested by Bewsher et al. (2010) is used to extract the nova light-curve from the data using aperture photometry. The solar F-corona is the dominant source in the HI-1B data, and is removed using a daily running background.

To extract the light-curve presented in this paper, each HI image is processed with SECCHI_PREP, and the position of the nova determined using the pointing information given in the header of the FITS file. Aperture photometry is performed on this position, and the number of counts (DN/s) recorded. This process is repeated for every HI image from 2012 April 18 to 2012 May 7, where the nova crosses the CCD of the instrument. The counts recorded are then converted into a magnitude using the following equation

$M_{H I}=-2.5 \log \left(\frac{I_{t o t}}{\mu F}\right)$

where $M_{H I}$ is the HI magnitude, $I_{t o t}$ is the recorded counts, $\mu$ is a calibration conversion factor and $F$ is the flux of Vega (Bewsher et al. 2010), the zero-point of the magnitude scale. The error on $\mu F, \sigma_{F}$, is estimated to be of the order of $5 \%$, therefore,

$\frac{\sigma_{F}}{\mu F}=0.05$

1 http://www.ukssdc.ac.uk/ 
It is assumed that the uncertainties on $I_{t o t}, \sigma_{I}$ are Poisson counting statistics, and the conversion from DN (units of $\left.I_{t o t}\right)$ to photoelectrons is $1 \mathrm{DN}=15$ photoelectrons (Eyles et al 2009), therefore,

$\sigma_{I}=\sqrt{\frac{I_{t o t}}{15}}$.

The uncertainty on the magnitude measurement is then given by

$$
\begin{aligned}
\sigma_{M_{H I}} & =\left|\frac{-2.5}{\ln (10)} \sqrt{\left(\frac{\sigma_{I}}{I_{\text {tot }}}\right)^{2}+\left(\frac{\sigma_{F}}{\mu F}\right)^{2}}\right| \\
& =\left|\frac{-2.5}{\ln (10)} \sqrt{\left(\frac{\sqrt{\frac{I_{t o t}}{15}}}{I_{\text {tot }}}\right)^{2}+(0.05)^{2}}\right|
\end{aligned}
$$

This value was calculated on a point-by-point basis and is included in Fig. 1 as error bars. The error due to the background subtraction has not been included in the errors on the light-curve. However, it is estimated that the error due to the background subtraction is of the order of $10 \%$ of the calculated error for a handful of points, and less than $5 \%$ for the majority of points. This is due to the background subtraction being calculated using the average over a days worth of observations leading to comparatively small uncertainties.

In the following we use the 40 min cadence of the data as the basis for uncertainties in any dates or intervals in time. This translates to $\pm 0.0139 \mathrm{~d}$, with the third decimal place being conservative given that observations are made at this cadence to within a few seconds.

\section{RESULTS}

The light-curve of V5589 Sgr is presented in Fig. 1. We see the rise to maximum, and then a rather erratic decline. We also overlay AAVSO B, V and visual estimate data for comparison. Uncertainties in AAVSO visual estimates are estimated as $0.1 \mathrm{mag}$ although this varies with location and experience of the contributing observers. We can see that while the STEREO and AAVSO data are consistent, the latter has a much lower cadence and relies primarily on visual estimates.

The time of maximum is a key characteristic determined for previous novae, and often used to refer to subsequent development, with the brightest point labeled as "day 0". As the decline time from peak is used to determine the speed class of CNe (Payne-Gaposhkin 1957; Duerbeck 2008), which in turn correlates with the energetics of the event, it is useful to identify this point. There is one brightest point in the STEREO data, albeit less than $1 \sigma$ above adjacent points. This point is at JD $2456038.8224 \pm 0.0139$, and can be taken as the peak magnitude.

Determining the time to decline by two magnitudes, $\mathrm{t}_{2}$, and hence the speed class, is somewhat more uncertain. The brightness at peak is $7.94 \pm 0.09$, and the brightness drops below 9.94 for the first time after JD $2456040.9057 \pm 0.0139$ but recovers $2 \mathrm{~h}$ later. The last time it is brighter than this is the point at JD 2456042.3224. The average magnitude binned over 18 points ( $12 \mathrm{~h}$ interval) reaches this level in the bin centred on JD 2456041.7668. These give a $t_{2}$ time of between 2.1 and 3.9 days, placing the nova firmly in the "very fast" class. The STEREO passband incorporated more red light than the $\mathrm{B}$ or $\mathrm{V}$ bands where $\mathrm{t}_{2}$ is usually determined. Nonetheless the values are well within the "very fast" range, and there is an expectation that this object will be in the upper range for intrinsic luminosity and velocity of the ejecta of the nova events. This is consistent with the identification of a high-speed shock by Weston et al. (2016).

\subsection{Pre--maximum halts}

A key finding of our work is the presence in the risephase of deviations from a monotonic increase that are consistent with $\mathrm{PMHs}$ seen in other classical novae. For V5589 Sgr we have identified two short deviations - peaking at JD $2456037.8502 \pm 0.0139$ and JD 2456037.9891 $\pm 0.0139-$ with this phenomenon. From Fig. 1 we can see that the rise to peak occurs between one point and the next (i.e. within $40 \mathrm{~min}$ ), with the decline over three points (i.e. over $80 \mathrm{~min}$ ), both with the same shape within the uncertainties. As the PMHs appear V5589 Sgr moves between pixels 80 and 110 along the 540th row of the CCD, so the nova is well away from the edge of the $1024 \times 1024$ pixel detector.

The initial rise reflects a change of more than $3 \sigma$ pointto-point as a PMH starts (uncertainties decline with increasing brightness). The PMH decline is to a level within $2 \sigma$ of the pre-rise magnitude. Modelling by Hillman et al. (2014) suggests that PMHs are often short-lived excursions above the general upwards trend of the rise. We explore this by taking linear interpolations between the "before" and "after" points for both features. These are found to follow a line consistent with the subsequent rise. Thus the two peaks are consistent with being such excursions from the general upwards trend, rather than being defined by dips that interrupt the rise. The combination of the change relative to the uncertainties and the deviation from what one would expect from backwards extrapolation of the rise, allows us to be confident that they can be reasonably described as PMHs as modelled by Hillman et al. (2014).

\section{DISCUSSION}

We discuss the pre-maximum halts during the rise phase, and then the behaviour following the optical peak.

\subsection{Double pre-maximum halt}

During the rise to maximum (Fig. 1), we see two very similar features that represent departures from the smooth, monotonic rise normally considered to be the usual behaviour for $\mathrm{CNe}$ before the optical peak. We have interpreted these as the sometimes-observed PMHs. When taken in combination with those seen by Hounsell et al. (2010) we can conclude that these events are common, and have been generally missed in historical observations.

In Fig. 2 we compare a model from the grid of Hillman et al. (2014) with the STEREO data. We have chosen parameters which give the best qualitative agreement with the data. We have not attempted to fit the parameters 


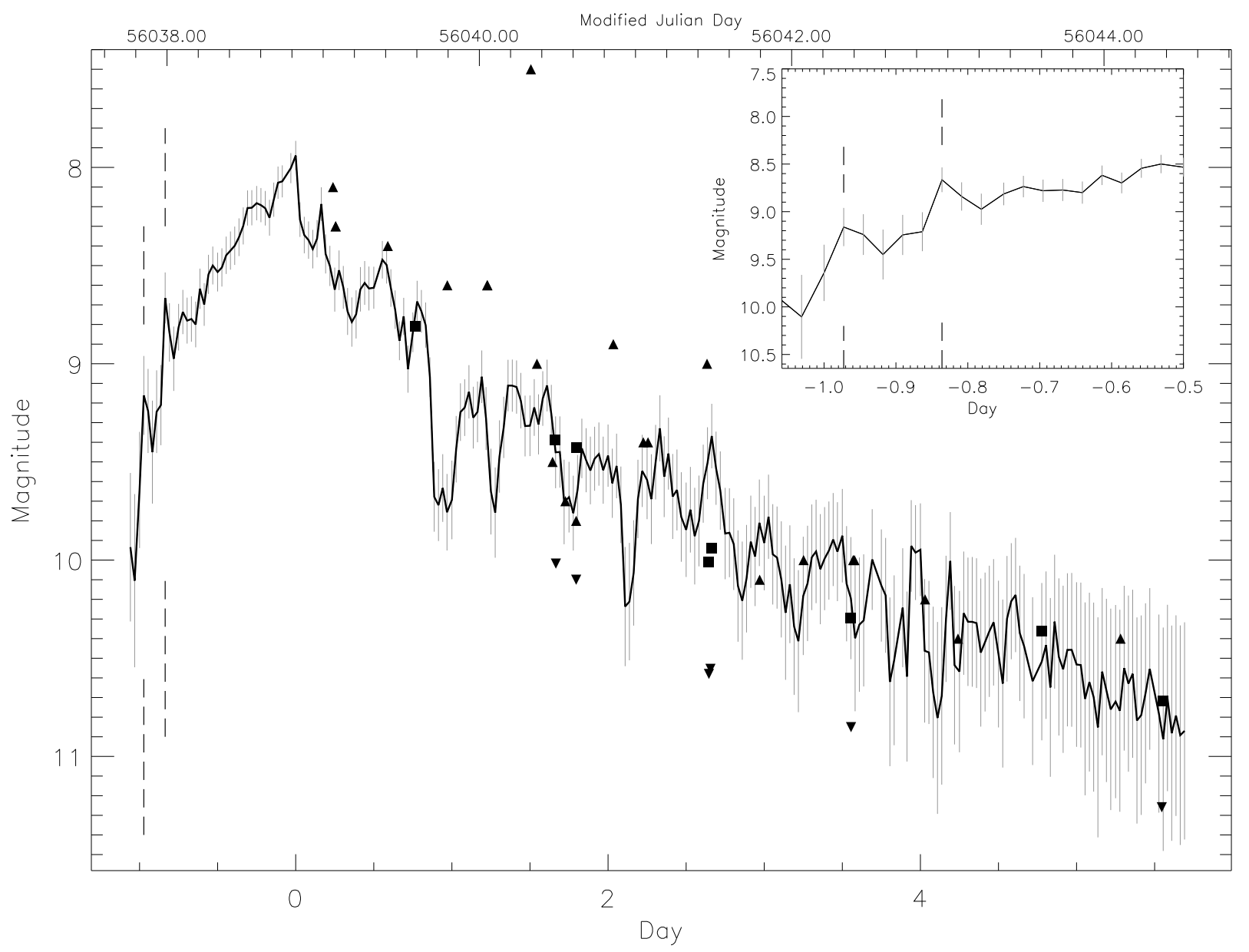

Figure 1. STEREO HI-1B data with error bars, plus AAVSO visual estimates (triangles), V (squares) and B (inverted triangles) included for comparison; offset by 1 mag upwards for clarity. STEREO is approximately R-band, for which data are available in AAVSO only after JD 2456051 (MJD 56051.5), about $6 \mathrm{~d}$ after the end of this data run. Vertical dashed lines mark the PMHs described in the text. The inset zooms in on the $\sim 14$ hour period around the PMHs.

to the data, but instead we offer this as support that the general development of the PMHs during the rise is understood. The model plot shows the relevant time period from Fig. 4, Panel 4, plot 100.50.10 of Hillman et al. (2014). The model starts from well before the span of the data, and to assess the similarity, we have simply aligned the peak of the second halt. By inspection we can then see that the duration, magnitude and interval between the halts is consistent between the model and the data. The PMHs occur in the model at the point when convection ceases to be efficient near the surface causing a reduction in the energy flux. The opacity then decreases allowing radiation to become the dominant energy transfer mechanism and reverse the dip. This starts to drive mass loss.

The model agrees less well with the behaviour of V5589 Sgr once convection ceases to dominate evolution around the PMHs. Nonetheless we have constrained the onset of mass loss to occur between the peak of the first $\mathrm{PMH}$ and the maximum of the optical light (i.e. between JD 2456037.8502 and JD 2456038.8224), with the model formally giving this as JD 2456038.2391. The modelling assumes a main-sequence donor star. While the subgiant donor would have a lower $\mathrm{H}$ abundance, this simply means the accretion rate derived from the model is an underestimate of the true rate. An optical spectrum in quiescence would be valuable to confirm the nature of the secondary.

\subsection{Post-maximum erratic decline}

There are a number of excursions visible in Fig. 1 signifcantly above or below the mean magnitude during the decline after day 0 . We consider if any of this structure is consistent with periodicity. We also examine how significant the excursions are given the overall scatter in the data.

An orbital period of $1.59230 \mathrm{~d}$ determined by Mróz et al. (2015) is based on the ephemeris of HJD = $2455000.724 \pm 0.015+(1.59230 \pm 0.00005) \times \mathrm{E}$ for the eclipses.

To test for periodicities in the decline phase, we excluded data obtained before day 0 and perform a discrete Fourier transform on the remaining data using the PERIOD04 software of Lenz \& Breger (2005). After fitting and removing the slope of the decline, we search for significant periods in the de-trended data. Given the quality of the observations, we relax the 'standard' S/N limit of 4 (e.g. Koen 


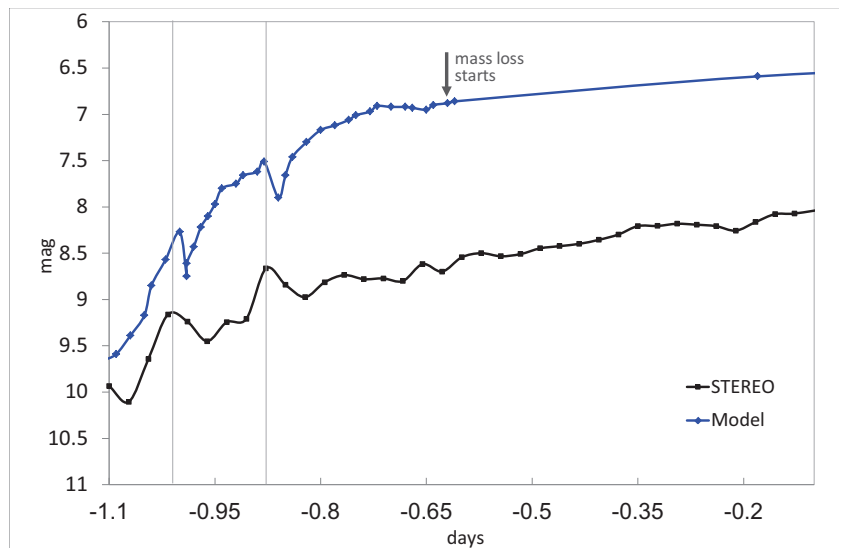

Figure 2. Comparison of data (lower line) and model (upper line) for the parameters giving the closest match to the pre-maximum behaviour (WD mass $1.0 \mathrm{M}_{\odot}$, central temperature $5 \times 10^{7} \mathrm{~K}$ and mass accretion rate $\left.10^{-10} \mathrm{M}_{\odot} \mathrm{yr}^{-1}\right)$. The time axis is relative to day 0 at JD 2456038.8224 .

2010) to 3 to determine if a peak in the FT is significant, however we find no peaks in the FT above this limit. Furthermore, there is no evidence in our data of the orbital period determined by Mróz et al. (2015).

This is consistent with post-outburst OGLE observations showing that eclipses do not start occuring again until HJD $=2456062.82544$ or possibly as late as HJD = 2456076.92252. Prior to this the entire binary must be within the photosphere of the nova ejecta, and so must be larger than the orbital radius of $\sim 7.2 R_{\odot} \times\left(M / 2 M_{\odot}\right)^{1 / 3}$ for a system mass of $M$.

While there are a number of variations in the decline phase, none are consistently separated by this interval, or a multiple thereof. In addition a simple polynomial fit to the decline suggests only the minima centred around MJD $56039.7668,56040.1002$ and 56040.9335 are consistent with anything other than the overall level of fluctuations seen. None of these are separated by the period in Mróz et al. (2015). Thus whatever the origin of these dips, neither eclipses nor orbital modulation appear to explain them.

The speed class appears to be related both to the luminosity of the nova at the peak, and the kinetic energy of the ejecta, with both being higher for faster novae. Much less consistently, more slowly declining examples often show erratic brightening and dimming after the initial rise, presenting multiple false maxima and making the determination of the peak, and hence the decline rate, difficult. Evans et al. (2003) discuss V723 Cas as an example, and suggest these "flare-like" features might be due to interactions within the ejecta or mass transfer bursts from the companion, as proposed by Chochol, O'Brien \& Bode (2000). By contrast erratic behaviour is not generally seen in fast examples of $\mathrm{CNe}$, where the rise and fall is seen to be smooth. However the vast majority of ground-based light-curves are made up of a number of observations every night, with considerable variation in the consistency of observations and are mainly visual estimates. The data presented here provide unprecedented stability and point-to-point reliability at a regular $40 \mathrm{~min}$ cadence.

Hounsell et al. (2010) found some erratic behaviour early on in V1280 Sco and to a lesser extent in KT Eri, the second also being very fast. As noted in their Section 4 the decline time and the form of the PMHs do not seem to have any correlation; V5589 Sgr shows a different form again even though the initial decline rate was similar to that of KT Eri. We suggest that the association of erratic brightness variations near peak with slower novae is a feature of the poor cadence of most light-curve data to date. It is clearly a feature of some examples of fast novae, but at this point we do not have enough examples to judge if it is less common than in slower novae.

In fact, once we start to record the early light-curve of novae at the cadence achieved here, the definition of "day 0 " needs to be revisited. An alternative approach to determining the peak in Fig. 1 is to treat all fluctations from a smooth rise and decline as anomalous. In this case some form of fit to the overall rise and decline might be used to give an estimate of the peak that is derived in a manner consistent with those for novae with only low cadence data. A second-order polynomial fit through the peak has a maximum of $8.16 \pm 0.10 \mathrm{mag}$, at 56038.68 , or 160 min earlier than the absolute brightest measurement used to define "day 0" in Section 4. Thus at this high cadence, where we see fluctations on timescales of a few hours, the relevance of optical maximum and hence "day 0" starts to become problematic. However as STEREO and other facilities allow us to examine an increasing number of fast novae in this detail, we may be able to reassess the speed class concept.

\section{CONCLUSIONS}

We have collected an optical light-curve of the fast Classical Nova V5589 Sgr at a time-resolution of $40 \mathrm{~min}$, the best for any nova to date. This data show the rise to maximum with pre-maximum halts, and the subsequent somewhat erratic decline, in greater detail than seen before. We find that the halts are consistent with a binary model having a WD of mass $1.0 \mathrm{M}_{\odot}$, central temperature $5 \times 10^{7} \mathrm{~K}$ and mass accretion rate $10^{-10} \mathrm{M}_{\odot} \mathrm{yr}^{-1}$. As the donor star is a subgiant, the last of these is likely an underestimate. The model shows the PMHs are pre-cursors to the onset of mass loss and formally predicts this as starting at JD 2456038.2391 \pm 0.0139 for V5589 Sgr.

We suggest that the erratic decline behaviour, a common characteristic of slow novae, is missed in previous observations of some faster examples by the typical daily observations. Our work, and that of Hounsell et al. (2010), demonstrate that some fast novae also demonstrate erratic behaviour close to optical peak. This is not understood or well modelled at this time. We also find that at such high cadence, the common identification of the peak as "day 0" becomes increasingly difficult and possibly misleading.

STEREO-A continues to operate and we will retrieve data of further $\mathrm{CNe}$ that are serendipitously bright as they cross one or more of the four camera apertures. This will allow us to investigate the prevalence and nature of PMHs, and any erratic behaviour close to optical maximum. 


\section{ACKNOWLEDGEMENTS}

Data created during this research is openly available from the University of Central Lancashire data repository, UCLanData, at http://doi.org/10.17030/uclan.data.00000049.

The Heliospheric Imager (HI) instrument was developed by a collaboration that included the Rutherford Appleton Laboratory and the University of Birmingham, both in the United Kingdom, and the Centre Spatial de Liége (CSL), Belgium, and the US Naval Research Laboratory (NRL), Washington DC, USA. The STEREO/SECCHI project is an international consortium of the Naval Research Laboratory (USA), Lockheed Martin Solar and Astrophysics Lab (USA), NASA Goddard Space Flight Center (USA), Rutherford Appleton Laboratory (UK), University of Birmingham (UK), Max-Planck-Institut für Sonnensystemforschung (Germany), Centre Spatial de Liège (Belgium), Institut d'Optique Théorique et Appliquée (France), and Institut d'Astrophysique Spatiale (France).

DLH acknowledges financial support from the STFC via grant ST/M000877/1.

\section{REFERENCES}

Ackermann M., et al., 2014, Sci, 345, 554

Bewsher D., et al., 2010, Solar Physics, 264, 433

Brown D.S., Bewsher D., Eyles C.J., 2009, Solar Physics, 254, 185

CBAT, 2015, "Transient Object Followup Reports" PNV J17452791-2305213, accessed 2015/10/27 http://www.cbat.eps.harvard.edu/unconf/followups/J17452791-2305213.html

Capaccioli M., della Valle M., D’Onofrio M., Rosino L., 1990, ApJ, 360, 63

Chochol D., O'Brien T. J., Bode M. F., 2000, NewAR, 44, P61

Darnley M. J., Bode M. F., Kerins E., et al., 2006, MNRAS, 369, 257

Downes R. A., Duerbeck H. W., 2000, AJ, 120, 2007

Duerbeck H. W., 2008, in: Classical Novae, Bode M. F., Evans A. (eds), pp1-15, Cambridge University Press, Cambridge

Esipov V. F., Sokolovsky K. V., Korotkiy S., 2012, ATel. 4094

Evans A., Gehrz R. D., Geballe T. R., et al., 2003, AJ, 126, 1981

Eyles, C.J., et al., 2009, Solar Physics, 254, 387

Hillman Y., Prialnik D., Kovetz A., Shara M. M., Neill J. D., 2014, MNRAS, 437, 1962

Holdsworth D.L., et al., 2014, MNRAS, 438, 3483

Hounsell R., et al., 2010, ApJ, 724, 480

Howard R. A., et al., 2008, Space Science Reviews, 136, 67

Kaiser. M.L., et al., 2008, Space Science Reviews, 136, 5

King A. R., 1989, in: Classical Novae, Bode M. F., Evans A. (eds), pp16-33, J. Wiley \& Sons, Chichester, UK

Koen C. 2010, Ap\&SS, 329, 267

Korotkiy S., et al. 2012, Central Bureau Electronic Telegrams, 3089, 1

Lenz P., \& Breger M. 2005, Communications in Asteroseismology, 146, 53

Mróz P., Udalski A., Poleski R., et al., 2015, ApJS, 219, 26

Nelson T., Mukai K., Sokoloski J., Chomiuk L., Rupen M., Mioduszewski, A., 2012a, ATel. 4088

Nelson T., Mukai K., Sokoloski J., Chomiuk L., Rupen M., Mioduszewski, A., 2012b, ATel. 4110

Payne-Gaposhki, C., 1957, The Galactic Novae. Amsterdam: North-Holland.

Sokolovsky K., Korotkiy S., Elenin L., Molotov I., 2012, ATel. 4061
Warner B., 2008, in: Classical Novae, Bode M. F., Evans A. (eds), pp16-33, Cambridge University Press, Cambridge

Weston J. H. S., et al., 2016, MNRAS, 460, 2687

Wraight K.T., et al., 2012, MNRAS, 426, 816

This paper has been typeset from a $\mathrm{T}_{\mathrm{EX}} / \mathrm{LAT}_{\mathrm{EX}}$ file prepared by the author. 